\title{
Toxic Epidermal Necrolysis Associated with Tetracycline Eye Ointment: A Case Report
}

Selamawit Gebrehiwet ${ }^{1}$, Abrahalei Kessete ${ }^{2}$, Mulugeta Russom ${ }^{1^{\star}}$ and Gelila Abrham ${ }^{3}$

${ }^{1}$ National Pharmacovigilance Centre, Asmara, Eritrea

${ }^{2}$ National Medicines and Food Administration, Asmara, Eritrea

${ }^{3}$ Tesseney Hospital, Tesseney, Eritrea

\begin{abstract}
Toxic epidermal necrolysis (TEN) is a rare but potentially life threatening severe cutaneous adverse reaction with widespread epidermal detachment and mucosal erosions usually induced by medications. Tetracycline eye ointment is not known to cause TEN and recent literature search found no published articles that associate tetracycline (TTC) eye ointment and TEN. However, the Eritrean National Pharmacovigilance Centre has received one serious cutaneous reaction immediately following application of TTC eye ointment. The aim of this case report is therefore to describe this unusual and interesting adverse drug reaction, it's possible explanations and treatment outcomes of the event. This is a case of 15 years old female patient admitted to hospital with a presentation of body skin desquamation, fever and malaise, eye and mouth pain with redness. Notably, this case study is designed for the peculiarity of its occurrence with a single dose instillation of tetracycline eye ointment purchased as an over the counter drug. As a result, patient developed generalized skin lesion with about $80 \%$ skin involvement. Though TEN is usually drug induced, it can also be explained with some etiological factors which can induce similar reaction such as infections as well as malignancy. Clinical and laboratory investigation report however show that the patient had no prior history of infection, history of allergy and other drugs intake. Taking into account the above facts complemented with the low disease background incidence, possible biological mechanism and the plausible time to reaction onset (4 days), this case is possibly associated with TTC eye ointment. The availability of tetracycline eye ointment without prescription in Eritrea makes this association alarming as it can amplify the occurrence of TEN. Hence, further studies are required to substantiate the association.
\end{abstract}

Keywords: Adverse drug reaction; Toxic epidermal necrolysis; Tetracycline eye ointment; Eritrea

Abbreviations: ALP: Alkaline Phosphatase; AST: Aspartate Aminotransferase; BP: Blood Pressure; CBC: Complete Blood Count; FBS: Fasting Blood Sugar; HIV: Human Immuno-deficiency; LFT: Liver Function Test; NSAIDs: Non-Steroidal Anti-inflammatory Drugs; PR: Pulse Rate; RFT: Renal Function Test; RR: Respiratory Rate; SPC: Summary of Product Characteristics; T: Temperature; TEN: Toxic Epidermal Necrolysis; TTC: Tetracycline

\section{Introduction}

Toxic epidermal necrolysis (TEN) is an acute-onset, potentially life-threatening, idiosyncratic mucocutaneous reaction, usually occurring with widespread full-thickness epidermal necrosis and sloughing of the skin and mucosa, involving internal and external surfaces [1]. The skin has an appearance similar to a scald. It usually affects the trunk, face and one or more mucous membranes. It occur sporadically more commonly in adults. TEN is a fairly rare event with an average incidence proportion of one case per million populations/ year [2,3]. An increased incidence has been observed in individuals with genetic variations, HLA-B 12 [4,5]. Drugs are the most common cause accounting for about $65 \%-80 \%$ of the cases $[2,6-10]$ and the most common offending agents are sulfonamides, NSAIDs, butazones and hydrantoins [2].

To the best of our knowledge, a recent literature search found no published articles, even case reports, that associate Tetracycline (TTC) eye ointment and TEN. Nevertheless, there is no mention of TEN as an adverse drug reaction in the Summary of Product Characteristics (SPC) or product information leaflets of TTC eye ointment [1114]. Moreover, search made on April 18, 2017 in the WHO Global adverse drug reaction database, VigiBaseTM, found no reports of TEN associated with TTC eye ointment. The Eritrean Pharmacovigilance
Centre however received a case of TEN following the use of TTC eye ointment. The aim of this case report is therefore to describe unusual and interesting adverse drug reaction in a patient treated with TTC eye ointment and its diagnosis, possible explanations and treatment outcomes of the adverse effect.

\section{Case Presentation}

A 15 years old female patient was admitted to Halibet National Referral Hospital with a complaint of malaise, fever, loss of appetite followed by generalized skin lesion all over the body of two days duration following a single application of tetracycline eye ointment. The medication was prescribed by a rural drug vender for eye infection three times a day for seven days. Following the first dose of TTC, the reaction manifested thus drug withdrawn immediately. Patient denies any history of drug and food allergy, had no history of exposure to other drugs, chemicals or herbal medicines. Up on rigorous investigation, patient had neither systemic disease nor has known infection.

During admission with the adverse drug reaction, patient was in distress, febrile but fully conscious with normal vital signs. Thorough physical examination revealed, bilateral desquamation of the eyelids,

*Corresponding author: Mulugeta Russom, Head, Eritrean Pharmacovigilance Centre, Asmara, Eritrea, Tel: +291-7197450; E-mail: satiswt@gmail.com

Received: December 12, 2017; Accepted January 17, 2018; Published January 24, 2018

Citation: Gebrehiwet S, Kessete A, Russom M, Abrham G (2018) Toxic Epidermal Necrolysis Associated with Tetracycline Eye Ointment: A Case Report. J Pharmacovigi 6: 251. doi:10.4172/2329-6887.1000251

Copyright: (c) 2018 Gebrehiwet S, et al. This is an open-access article distributed under the terms of the Creative Commons Attribution License, which permits unrestricted use, distribution, and reproduction in any medium, provided the original author and source are credited. 
with conjuctival hyperemia and edema. Multiple erosion and crusts were present on the lips, buccal mucosa with swollen and tender tongue. The patient had also experienced a widespread Erythema and confluent vesiculation leading to desquamation and sloughing of the skin over the face, neck, back, abdomen, buttock, genitals and extremities. Nikolsky sign (i.e, the ability to extend the area of superficial sloughing by gentle lateral pressure on the surface of the skin at an apparently uninvolved site) was positive.

Greater than eighty percent of the total body surface area was affected by the severe cutaneous reaction. Investigations including complete blood count (CBC), random blood sugar (RBS), Urinalysis, liver function tests (LFT), Lipid panel, renal function tests (RFT) and electrolytes were done to identify possible causes and the results were as follows: Alanine aminotransferase (ALT): $125 \mathrm{IU} / \mathrm{L}$, aspartate aminotransferase (AST): $79 \mathrm{IU} / \mathrm{L}$, alkaline phosphatase (ALP): $130 \mathrm{IU} / \mathrm{L}$; cholesterol $273 \mathrm{mg} / \mathrm{dl}$; RBS $132 \mathrm{mg} / \mathrm{dl}$, HIV negative. In contrast to the above results, complete blood count (CBC), fasting blood sugar (FBS), urinalysis, bilirubin and electrolyte function were all normal. Renal function test (RFT) revealed very low serum creatinine 0.21 . Management of the patient was mainly focused in the immediate removal and discontinuation of the tetracycline eye ointment and admission for supportive care. Patient was on maintenance IV fluids, nutritional support with naso-gastric tube, intravenous hydrocortisone injection with loading dose of 200 $\mathrm{mg}$ and maintenance dose of $100 \mathrm{mg}$ bid followed by tapered dose of prednisolone for one week. Besides, wound care with antiseptic solution and dressing with neomycin+bacitracin ointment as well as betamethasone+salicylic acid on daily bases were done. Two weeks after admission, general condition of the patient improved and the patient's skin was healing rapidly with some scars. On discharge, patient was carefully advised about the future avoidance and potential seriousness of re-exposure to the suspected drug. Delayed ophthalmologic examination (three months later) also revealed that there is presence of eye infection with symptoms of itching upon exposure to sun-light.

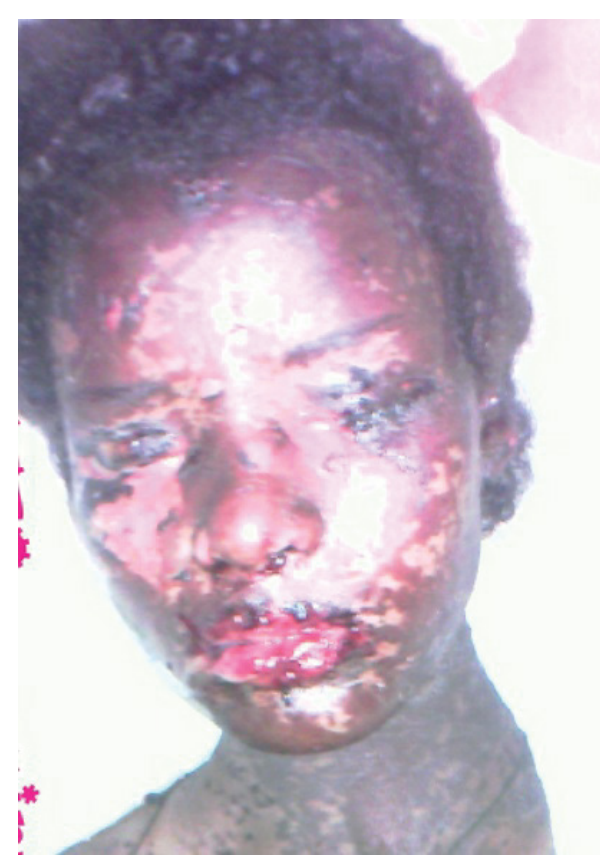

Figure 1: Toxic Epidermal Necrolysis.

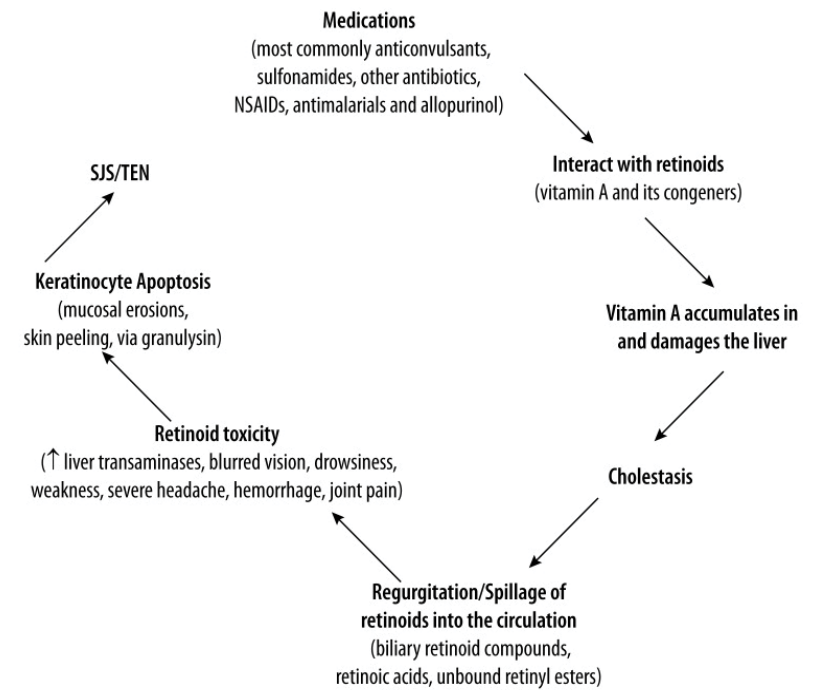

Figure 2: Hypothesis on mechanism of drug-induced SJS/TEN by interacting with retinoids.

\section{Discussion and Conclusion}

Based on the patient's medical history, percentage of the affected body surface area, skin detachment with the involvement of mucous membranes and early signs and symptoms, a final diagnosis of toxic epidermal necrolysis secondary to tetracycline eye ointment was considered. Though TEN is usually drug-induced, it can also be explained with some etiological factors which can induce the reaction such as infections as well as malignancy. Clinical and laboratory investigation report however show that the patient had no prior history of infection, history of allergy and other drugs intake. Taking into account the above facts complemented with the low disease background incidence [1] and the plausible time to reaction onset (4 days), this case is possibly associated with TTC eye ointment. Tetracycline tablet [15] was previously associated with Stevens-Johnson syndrome and toxic epidermal necrolysis (SJS/TEN) that also raises the established suspicion.

The possible mechanism of medication-induced SJS/TEN is by interacting with endogenous retinoids (vitamin A and its congeners), in many instances causing the latter to accumulate in and cause inhibition of cell growth or cytotoxicity, mutagenicity, and teratogenicity and can also damage the liver, the main storage organ for vitamin A [15-17]. The hypothesis is that the cholestatic liver damage leads to the spillage of toxic retinoid compounds into the circulation, resulting retinoid toxicity which eventually lead to keratinocyte apoptosis (cell death) mediated by granulysin and recognized as SJS/TEN [17] (Figure 1, 2). Tetracycline is known to cause inhibition of cytochrome P450mediated degradation [17] of retinoic acids which can lead to retinoid toxicity and might end up with keratinocyte apoptosis or SIS/TEN (Figure 1). This might be the cause that the liver enzymes in our patient were moderately elevated. The availability of tetracycline eye ointment without prescription in Eritrea makes this association alarming as it can amplify the occurrence of TEN. Hence, further studies are required to assess the causal association between tetracycline eye ointment and toxic epidermal necrolysis.

\section{Key messages}

- Consumers should be aware on the early signs and symptoms of TEN which can tighten/reduce the complications by taking action as early as possible. 
Citation: Gebrehiwet S, Kessete A, Russom M, Abrham G (2018) Toxic Epidermal Necrolysis Associated with Tetracycline Eye Ointment: A Case Report. J Pharmacovigil 6: 251. doi:10.4172/2329-6887.1000251

- Information on previous history of allergy related to TTC should be taken before prescribing tetracycline eye ointment.

- Long-term sequelae of the skin and eyes are common among survivors of TEN. Hence, early ophthalmic and dermatologic consultations and follow-ups have paramount importance.

- Prolonged use of corticosteroids as management for TEN should be discouraged as they can aggravate the condition.

- Healthcare professionals and patients should report encountered adverse drug reactions at the earliest possible time.

\section{Authors' Contribution}

All the authors contributed in the assessment, investigation, writeup and editing of the manuscript and give their consent for publication.

\section{Ethical Considerations}

Written consent was obtained from the family of the case the event to be shared locally and internationally. Patient identifiers like her name and place of residence/village are deanonymised in the publication.

\section{Competing Interests}

The authors declare that they have no competing interests and no source of funding was used to carry out the investigation.

\section{Acknowledgements}

The authors would like to acknowledge Halibet National Referral Hospital for allowing us to investigate the case. We would also like to sincerely thank the family of the case to allow us the event to be shared locally and internationally.

\section{References}

1. Schwartz RA, McDonough PH, Lee BW (2013) Toxic epidermal necrolysis: Part I. Introduction, history, classification, clinical features, systemic manifestations, etiology and immunopathogenesis. J Am Acad 69: 173 e1-173 e13.
2. Roujeau JC, Guillaume JC, Fabre JP, Penso D, Flechet ML, et al. (1990) Toxic epidermal necrolysis (Lyell syndrome) incidence and drug etiology in France. Arch Dermatol 126: 37-42.

3. Schopf E, Stuhmer A, Rzany B, Victor N, Zentgraf R, et al. (1991) Toxic epidermal necrolysis and Stevens-Johnson syndrome. An epidemiologic study from West Germany. Arch Dermatol 127: 839-842.

4. Yip VL, Alfirevic A, Pirmohamed M (2014) Genetics of immune-mediated adverse drug reactions: A comprehensive and clinical review. Clin Rev Allergy Immunol 48: 165-175.

5. Becquemont L (2010) HLA: A pharmacogenomics success story Pharmacogenomics 11: 277-281.

6. Sassolas B, Haddad C, Mockenhaupt M, Dunant A, Liss Y, et al. (2010) ALDEN an algorithm for assessment of drug causality in Stevens-Johnson syndrome and toxic epidermal necrolysis: Comparison with case-control analysis. Clin Pharmacol Ther 88: 60-68.

7. Valeyrie AL, Roujeau JC (2008) Epidermal Necrolysis Stevens-Johnsons syndrome and toxic epidermal necrolysis Fitzpatrick's Dermatology in General Medicine. McGraw-Hill. pp: 349-355.

8. Mockenhaupt M (2009) Severe cutaneous adverse reactions. Braun-Falco's Dermatology: 473-484

9. Breathnach SM (2010) Erythema multiforme, Stevens-Johnson syndrome and toxic epidermal necrolysis. Wiley-Blackwell.

10. IVAX Corporation. Tetracycline Hydrochloride, Miami, FL.

11. Lederle Laboratories. Achromycin, Wayne, NJ.

12. https://www.drugs.com/sfx/tetracycline-topical-side-effects.htm

13. https://www.medicines.org.uk/emc

14. Electronic Medicines Compendium (2016) Summary of Product Characteristics of Tetracycline Tablets BP $250 \mathrm{mg}$. Intrapharm Laboratories Limited.

15. Ganguly J (1989) The Biochemistry of Vitamin A. Boca Raton.

16. Hoffman C, Eichele G (1994) Retinoids in development. The Retinoids: Biology, Chemistry and Medicine. pp: 387-441.

17. Anthony RM, Ike E, Sridhar K (2015) Stevens-Johnson syndrome and toxic epidermal necrolysis (SJS/TEN): Could retinoids play a causative role. Med Sci Monit 21: 133-143. 